\title{
Correlation between Serum Anti-Müllerian Hormone (AMH) Level in Women and Follicular Response in the First In Vitro Fertilization (IVF) Cycle in Predict Live Birth from One Stimulation Cycle
}

\author{
Moubarak H, Mokdad M, Farhat S, Fakih C and \\ Darido J* \\ Darido J, Saint Georges University Hospital, Beirut, \\ Lebanon \\ *Corresponding author: Darido J , Saint Georges \\ University Hospital, Beirut, Lebanon
}

Received: May 11, 2018; Accepted: J une 08, 2018; Published: June 11, 2018

\begin{abstract}
Background: In vitro fertilization, a form of assisted reproductive technique, is the union of a woman's egg and a man's spermatozoid outside the body. A number of parameters known as ovarian reserve markers, such as serum Follicle Stimulating Hormone (FSH) concentration, Antral Follicle Count (AFC) and serum anti-Müllerian hormone (AMH) concentration are widely used to predict ovarian responses to gonadotrophin stimulation during in vitro fertilization treatment. To obtain satisfactory results, it is necessary to assess ovarian reserve before planning treatment.
\end{abstract}

Methods: This is a retrospective study to evaluate the role of $\mathrm{AMH}$ in the prediction of ovarian response following an ovarian stimulation protocol in the assisted reproductive technologies cycles. Follow- up of 100 patients admitted to the IVF center of Mount Lebanon Hospital through year 2012.

Results: Retrospective analysis of data collected from the clinic of the doctor and the IVF center showed a positive association between ovarian response in terms of total number of oocytes and anti-Müllerian hormone levels. Higher level of AMH was associated with increased risk of developing hyperstimulation ovarian syndrome in response to IVF drugs.

Conclusion: Serum AMH levels confirmed as a marker for ovarian reserve and ovarian response during IVF also for individualization of both the treatment strategy and the patient's expectations with a superiority on the other parameters.

Keywords: Anti-Müllerian hormone; Controlled ovarian stimulation; Follicular fluid; Ovarian hyperstimulation syndrome

\section{Abbreviations}

AFC: Antral Follicle Count; AMH: Anti-Müllerian Hormone; ART: Assisted Reproductive Technology; AUC: Area under the Curve; COS: Controlled Ovarian Stimulation; E2: Estradiol; ET: Embryo Transfer; FF: Follicular Fluid; FSH: Follicular Stimulating Hormone; HCG: Human Chorionic Gonadotropin; HFEA: Human Fertilization and Embryology Authority; ICF: In Vitro Fertilization; ICSI: Intracytoplasmic Sperm Injection; INH-B: Inhibin-B; LBR: Live Birth Rate; MLH: Mount Lebanon Hospital; OHSS: Ovarian Hyperstimulation Syndrome; PCOS: Polycystic Ovarian Syndrome; rLH: Recombinant Luteinizing Hormone; EGF: Vascular Endothelial Growth Factor

\section{Introduction}

\section{Overview of infertility: definition, prevalence, causes and treatment options}

Infertility is defined by the failure to achieve a pregnancy after 12 months of regular unprotected sexual intercourse, although the criteria for the duration vary between different countries.
Worldwide prevalence of infertility is estimated to be around 72.4 million couples and around 40 million of those seek medical care [1]. In the UK, 15\% couples present with infertility with an annual incidence of 1.2 couples per 1000 general population [2]. The main causes of infertility are tubal disease, ovulatory disorders, male factor and poor ovarian reserve. In a third of couples the cause of failure to achieve pregnancy is not established which is known as unexplained infertility (NICE 2013). Effective treatment options include improving lifestyle factors, medical and/or surgical treatment of underlying pathology, induction of ovulation and Assisted Reproductive Technology (ART). Assisted Reproduction consists of intrauterine insemination (IUI) and in vitro fertilization (IVF) cycles with or without intracytoplasmic sperm injection (ICSI) as well as treatment involving donated gametes.

\section{Overview of IVF}

In vitro fertilization, a form of assisted reproductive technique, is the union of a woman's egg and a man's spermatozoid outside the body.

An IVF treatment cycle involves: a) pituitary down-regulation;
Austin J Obstet Gynecol - Volume 5 Issue 6 - 2018

Submit your Manuscript | www.austinpublishing group.com

Darido et al. (C) All rights are reserved
Citation: Moubarak H, Mokdad M, Farhat S, Fakih C and Darido J. Correlation between Serum Anti-Müllerian Hormone (AMH) Level in Women and Follicular Response in the First In Vitro Fertilization (IVF) Cycle in Predict Live Birth from One Stimulation Cycle. Austin J Obstet Gynecol. 2018; 5(6): 1117. 


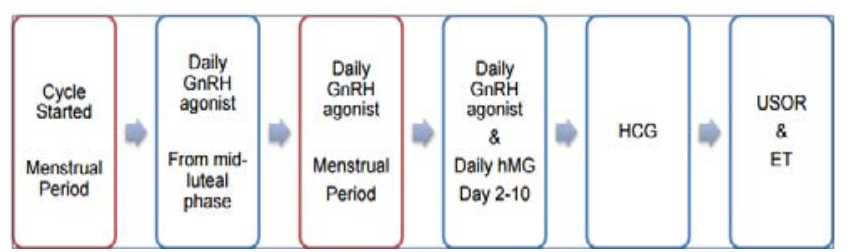

Figure 1: Schematic representation of a long $\mathrm{GnRH}$ (gonadotrophin releasing hormone) Agonist Cycle [3].

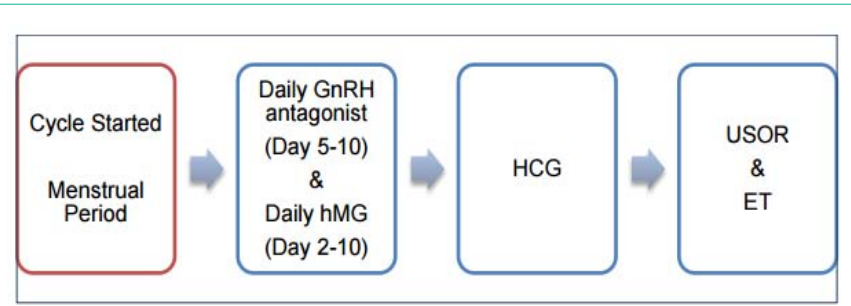

Figure 2: Schematic representation of $\mathrm{GnRH}$ (gonadotrophin releasing hormone) Antagonist Cycle [3].

b) controlled ovarian stimulation; c) oocyte recovery; c) in vitro fertilization of eggs with sperm; d) transfer of resulting embryo(s) back to uterus and e) luteal phase support. Prevention of premature surge of luteinizing hormone during controlled ovarian stimulation (COS) is achieved by pituitary down-regulation using either preparations of gonadotrophin releasing hormone agonist, which is widely known as "Agonist cycle" or gonadotrophin releasing hormone antagonist which is known "Antagonist cycle" (Figure 1 and 2). Controlled ovarian stimulation involves administration of gonadotrophins to encourage the development of supernumerary preovulatory follicles followed by administration of exogenous human chorionic gonadotropin (hCG) or recombinant luteinizing hormone $(\mathrm{rLH})$ to assist in maturation of oocytes 34-36 hours prior to egg collection which is usually conducted with guidance of transvaginal ultrasound scanning. Subject to sperm parameters, the fertilization of oocytes is conducted by in vitro insemination or intracytoplasmic sperm injection. The resulting embryo(s) are cultured under strict laboratory conditions and undergo regular qualitative and quantitative assessments before transferring the best quality embryo(s) back into uterus, during their cleavage (Day 2 or Day 3) or blastocyst (Day 5 or Day 6) stage of development. In natural menstrual cycles, under the influence of HCG, progesterone secreted by the ovarian corpus luteum ensures proliferative changes in the endometrium providing the optimal environment for implantation of embryo(s). However, in IVF treatment cycles, owing to pituitary down regulation and lack of HCG, progesterone levels are not in sufficiently high concentration to ensure an adequate endometrial receptivity. Therefore, exogenous analogues of this hormone are administered following transfer of embryo(s). This is called "luteal phase support" which, in patients with viable pregnancy, usually lasts till $12^{\text {th }}$ week of gestation, when the placenta starts producing progesterone in sufficient quantities [3].

\section{Parameters that correlate with the success of IVF}

In IVF programs, the "success" of the treatment is often defined as achieving a live birth following IVF cycle. This success is expressed using Live Birth Rate (LBR). In general, success in IVF is predominantly determined by the woman's age, cause(s) of infertility, ovarian reserve, previous reproductive history and lifestyle factors. However, effectiveness of medical interventions as well as the quality of care play important role in determining the outcome of IVF treatment. This is evident from significant variation in live birth rates among fertility clinics. For instance, in the UK, LBR for women younger than 35 years of age after one or more IVF cycles varies from $15 \%$ to $61 \%$.

According to the Human Fertilization and Embryology Authority (HFEA) data, around $12 \%$ of IVF cycles are cancelled due to poor or excessive ovarian response. Availability of reliable markers for assessment of ovarian reserve and tailoring ovarian stimulation regimens to the need of each individual patient may improve selection of patients with sufficient ovarian reserve and reduce the rate of cycle cancellation, consequently improving the success of IVF cycles.

\section{Parameters that predict ovarian reserve}

Assessment of ovarian reserve can be achieved using various biomarkers and four of those are currently used by most clinics: woman's chronological age (Age), serum follicle stimulating hormone (FSH), antral follicle count (AFC) and serum anti-Müllerian hormone (AMH).

\section{Anti-müllerian hormone (AMH)}

$\mathrm{AMH}$, also known as Müllerian-inhibiting substance is a dimeric glycoprotein that belongs to the transforming growth factor-beta family. It is involved in the regression of the Müllerian ducts during development of the male fetus.

AMH and folliculogenesis: AMH is produced by granulosa cells in primordial preantral and small antral follicles. The production of $\mathrm{AMH}$ in the follicle starts from the moment of its recruitment and lasts to antral stage development. The highest level of hormone synthesis is observed in granulosa cells of preantral and small antral follicles. AMH inhibits gene and protein expression of cytochrome P450 in aromatase granular cells [4]. By reducing the effect of FSH on preantral and antral follicle growth, the anti-Müllerian hormone participates in the regulation of folliculogenesis, thereby inhibiting the recruitment of germinal vesicles.

AMH as a marker of female fertility: During a woman's life, serum AMH concentrations are low after birth and during the prepubertal phase. At puberty, AMH concentration in the blood increases, peaking at approximately 20-25 years of age. After this time, hormone concentrations decrease to undetectable levels following menopause. During one menstrual cycle, hormone levels are not significantly affected [5]. Various factors have been reported to influence differences in AMH concentrations: ethnicity, smoking, vitamin D levels, and obesity. It was found that African-American and Spanish women have lower values of AMH than Caucasian women. Also, women who smoke present a lower level of anti-Müllerian hormone than non-smokers [6] (Figure 3).

AMH and Polycystic Ovary Syndrome (PCOS): It has been observed that in women with PCOS syndrome, the level of AMH is 2-3 times higher than in healthy women with normal ovulation [7]. A growing number of studies suggest that the diagnosis of PCOS, which is now determined using an ultrasound evaluation of the ovaries, anovulatory cycles, and hyperandrogenemia, should be replaced by the measurement of AMH [7]. 


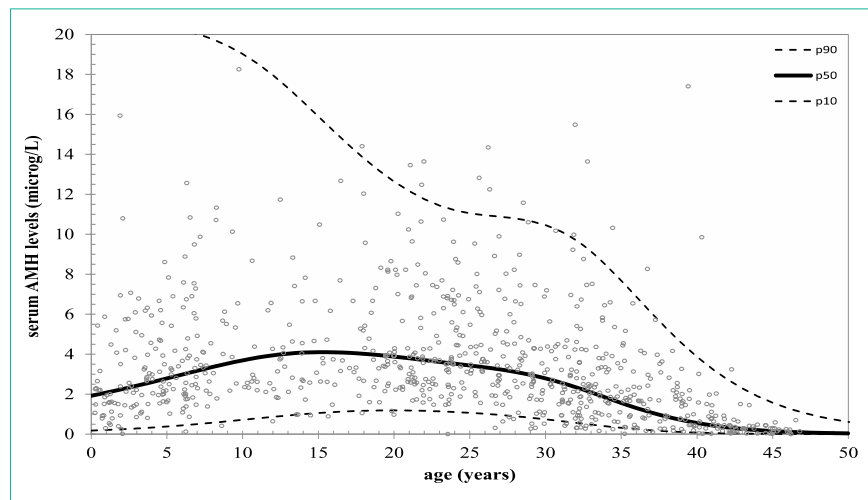

Figure 3: $\mathrm{AMH}$ nomogram based on natural linear spline interpolation Reference lines of serum $\mathrm{AMH}$ for the $10^{\text {th }}, 50^{\text {th }}$, and $90^{\text {th }}$ percentiles of predicted $\mathrm{AMH}$ values vs. Age [6]

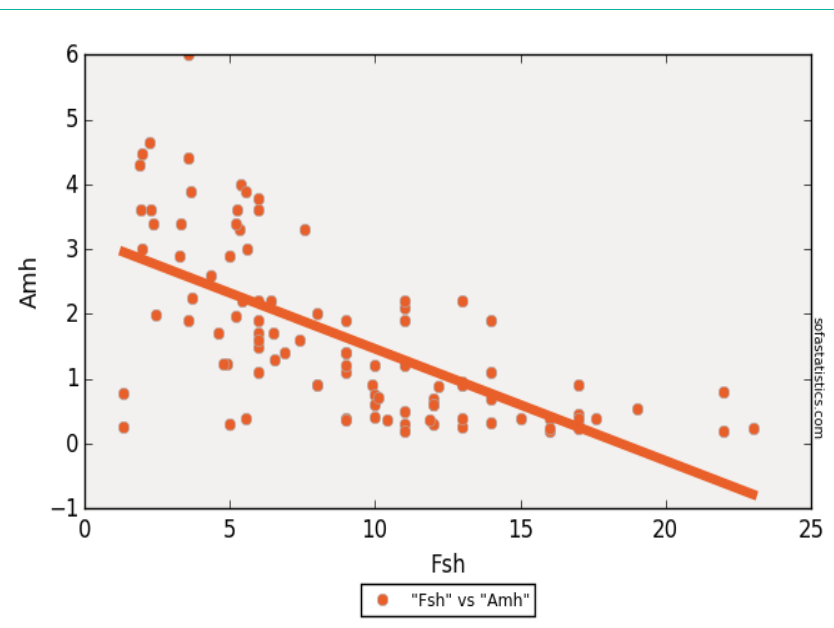

Figure 4: Linear regression of $\mathrm{AMH}$ with $\mathrm{FSH}$.

\section{Ovarian hyperstimulation syndrome: a complication of IVF}

Ovarian hyperstimulation syndrome (OHSS) is an iatrogenic complication of assisted reproduction technology. The syndrome is characterized by cystic enlargement of the ovaries and a fluid shift from the intravascular to the third space due to increased capillary permeability and ovarian neoangiogenesis. Its occurrence is dependent on the administration of human chorionic gonadotrophin (hCG). OHSS is extremely rare without hCG administration. Its impact on the general health of the patient can be very deleterious and fatal cases have occasionally been reported. The relationship between hCG and OHSS is thought to be mediated via the production of the angiogenic molecule VEGF. The incidence of moderate OHSS is estimated to be between $3 \%$ and $6 \%$, while the severe form may occur in $0.1-3 \%$ of all cycles [2].

Pathophysiology of OHSS: The processes are related to increased vascular permeability in the region surrounding the ovaries and their vasculature.

Clinical features of OHSS: Enlargement of the ovaries causes abdominal pain, nausea and vomiting. The enlargement is sometimes as much as $25 \mathrm{~cm}$ [8]. Acute abdominal pain may be due to ovarian torsion, intraperitoneal hemorrhage or rupture of cysts.
Another consequence is discomfort resulting from increased intraabdominal pressure due to ascites. Leakage of fluid from follicles, increased capillary permeability leading to third spacing (due to the release of vasoactive substances), or frank rupture of follicles can all cause ascites. Localized or generalized peritonitis is caused by peritoneal irritation secondary to blood from ruptured cysts, protein rich fluid and inflammatory mediators. Due to leakage of fluid through the impaired blood vessels both within and outside the ovary there is massive fluid-shift from the intravascular bed to the third compartment, which results in intravascular hypovolemia and hypotension with concomitant development of edema, ascites, hydrothorax and/or hydro pericardium. Pulmonary function may be compromised as enlarged ovaries and ascites restrict diaphragmatic movement. Hypercoagulable state due to hemoconcentration and hypovolemia resulting from fluid to third space shift. Patients have an increased risk of developing deep venous thrombosis and pulmonary embolism. Hypovolemia of OHSS leads to hemoconcentration and creates a hypercoagulable state. Microthrombi in renal tubules lead to decreased renal perfusion. Acute renal failure may result.

\section{Classification of severity of OHSS [9]:}

\section{Mild OHSS:}

Grade 1: Abdominal distention and discomfort.

Grade 2: Grade 1 disease plus nausea, vomiting, ovarian enlargement from $5-12 \mathrm{~cm}$.

\section{Moderate OHSS:}

Grade 3: Features of mild OHSS plus ultra-sonographic evidence of ascites.

\section{Severe OHSS:}

Grade 4: Features of moderate OHSS plus clinical evidence of ascites and/or hydrothorax and breathing difficulties.

Grade 5: All of the above plus a change in the blood volume, increased blood viscosity due to hemoconcentration, coagulation abnormalities and diminished renal perfusion and function.

Treatment of OHSS: Mild cases of OHSS can be treated with observation, bed rest, provision of adequate fluids and sonographic monitoring of the size of cysts. Serum electrolyte concentrations, hematocrit and creatinine levels should also be evaluated. The beginning of the resolution of OHSS is apparent when the cysts shrink, as seen on two consecutive ultrasonographic examinations and when clinical symptoms recede. In contrast, early detection of progression to the severe form of the syndrome is marked by continuous weight gain, increased severity of existing symptoms, or appearance of new symptoms. Medical treatment of severe hyperstimulation is directed at maintaining intravascular blood volume. Simultaneous goals are correcting the disturbed fluid and electrolyte balance, relieving secondary complications of ascites and hydrothorax and preventing thromboembolic phenomena (administration of heparin). To manage ascites, ultrasonographic-guided paracentesis is indicated if the patient has severe discomfort or pain or if she has pulmonary or renal compromise.

\section{Review of Literature}

More recently, $\mathrm{AMH}$ has been a focus of interest, given it 
is the only available endocrine marker that is suitable for direct assessment of the activity of ovarian follicles in their noncyclical stage development, providing a window to FSH independent phase of follicular recruitment. Furthermore, it appears to be reliable biomarker for:

- Both the assessment of ovarian reserve and the optimization of ovarian stimulation regimens $[10,11]$.

- Screening and diagnosis of polycystic ovarian syndrome (PCOS) [12].

- Monitoring of disease activity in women with a history of granulosa cell tumors [13]

- Prediction of the age of diminished fertility and menopause $[14,15]$.

- Assessment of the long term effect of chemotherapy on ovarian reserve [16].

Since AMH performs equally well, if not better, then AFC in predicting ovarian response and that it is both operator-and menstrual cycle-independent, there has been a growing trend to adopt $\mathrm{AMH}$ assay as the first line ovarian reserve test.

The secretion of AMH over the reproductive cycle of the women is as the follow: it begins at the perinatal period, increases around the time of puberty to maximal concentrations in early adulthood. Thereafter, a steady decline is seen until AMH becomes undetectable in the circulation several years prior to the menopause.

Multiple studies show that serum AMH showed a close correlation with the number of oocytes that were obtained following superovulation for IVF, and that serum AMH level also declines with age in women (age-specific interpretation of AMH).

In a retrospective study designed to evaluate the correlation between chronological and biological age by comparing the nomograms of AFC, AMH, and FSH: results were as follow: first the antral follicle counts and serum AMH decreased with increasing age, whereas serum FSH actually increased with advancing age. Second $\mathrm{AMH}$ and FSH Changed linearly. Third the change in serum AMH occurs earlier than in AFC or serum FSH. Therefore, serum AMH is an earlier predictor for biological age [17].

Another retrospective study that evaluates relationship between serum $\mathrm{AMH}$ and the rate of ongoing pregnancy in first embryo transfer cycles showed that serum AMH measurements reflect the total pool of AMH-producing follicles. Serum AMH level is closely related to the ovarian reserve and might be of secondary importance for ongoing pregnancy, with the most important factor for pregnancy being age. High serum AMH could be predictive of the presence of high-quality embryos on D3 and could potentially improve the rates of embryo development to the blastocyst stage, (single) blastocyst transfer, and pregnancy. Reductions in serum AMH due to ovarian aging reflects not only a reduction in the size of the primordial follicle pool but also an increasing rate of per-follicle granulosa cell apoptosis, which would be expected to reduce the per-follicle production of $\mathrm{AMH}$ and diminish oocyte quality [18].

One retrospective study examines common clinical determinants, including patient age; levels of anti-Müllerian hormone (AMH),

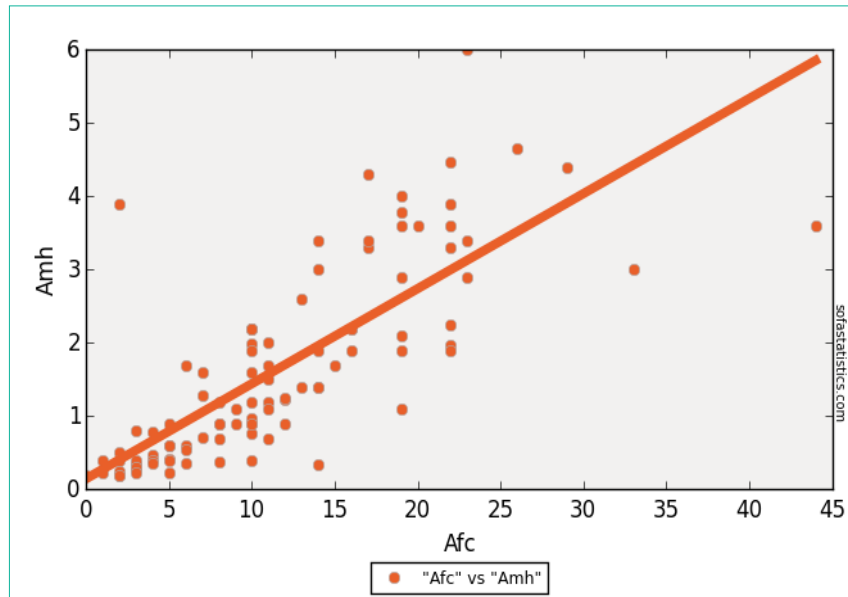

Figure 5: Linear regression of AMH with AFC.

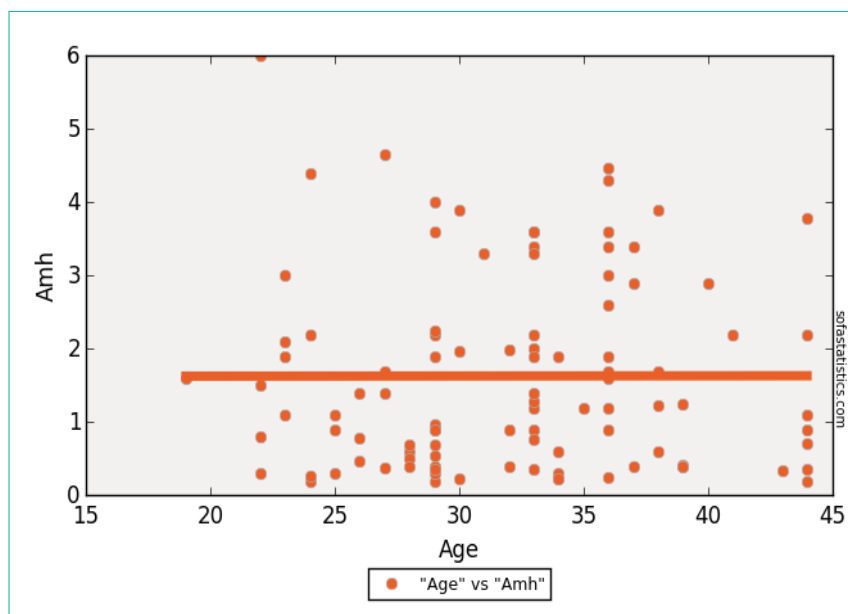

Figure 6: Linear regression of $\mathrm{AMH}$ with Age.

inhibin $\mathrm{B}$, and follicle-stimulating hormone (FSH), antral follicle count (AFC) and number of oocytes retrieved, to predict live births in women undergoing in vitro fertilization. Statistical analysis revealed that the odds of live birth significantly decreased with increasing age, declining $\mathrm{AMH}$ or inhibin B concentrations, and fewer oocytes retrieved. At higher AMH levels, the odds of live birth were greater than for lower AMH levels. Only AMH and AFC showed statistically significant associations with live birth. In this assessment of various indices (age; levels of $\mathrm{AMH}$, inhibin $\mathrm{B}$, and $\mathrm{FSH}$; $\mathrm{AFC}$; and quantity of oocytes retrieved) for predicting live births for IVF patients; AMH, AFC and the quantity of oocytes retrieved constituted the most reliable determinants [19].

Another prospective study performed on patients undergoing their first IVF trial, showed that AMH is a promising biochemical marker for the prediction of ovarian response and that a cut-off point indicating the value of $2.97 \mathrm{ng} / \mathrm{ml}$ can be adopted for this prediction [20].

Concerning ovarian hyperstimulation syndrome and its correlation with serum AMH level, several studies showed that mean AMH levels in serum and in FF were significantly higher in patients with OHSS than in normal patients [21]. 
Regarding the biological effect of $\mathrm{AMH}$, subsequent studies have indicated that it may regulate the responsiveness of growing follicles to FSH [22].

Other clinical applications of AMH include prediction of premature ovarian failure and menopause (Prediction of reproductive lifespan), diagnosis of polycystic ovary syndrome, prediction of poor response in IVF, prediction of ovarian hyper stimulation in IVF, detection of Ovarian damage post chemotherapy, radiotherapy and ovarian surgery.

In conclusion the review of literature revealed that $\mathrm{AMH}$ levels are a sensitive parameter for the prediction of response to ovarian stimulation with gonadotropins. The levels can be used as a tool for pre-stimulation patient counseling regarding the expected ovarian response (poor, moderate and high) and outcome (pregnancy rate, OHSS and cycle cancelation).

\section{Objectives}

\section{Main objective}

In our study, we focused on the role of AMH in the prediction of ovarian response following an ovarian stimulation protocol in the assisted reproductive technologies cycles, through searching a correlation between AMH levels in serum and the number of recruited mature follicles after an ovarian stimulation protocol.

\section{Secondary objectives}

Our study also intended to show the superiority of $\mathrm{AMH}$ in prediction of ovarian response on the other parameters especially FSH. We intended to show the relation between serum AMH level and occurrence of ovarian hyperstimulation syndrome.

\section{Materials and Methods}

\section{Study Design and Population}

Our study pretends to follow all patients admitted to Mount Lebanon Hospital (MLH) IVF center through year 2012.

Our study population included around 100 patients.

The study was a retrospective analysis of data collected from the clinic of the doctor and the IVF center

\section{Inclusion criteria:}

- Infertile women undergoing the first IVF stimulation cycle in MLH through year 2012.

- Regular menstrual cycle.

- $\quad$ Not on hormone therapy for three months.

- Have not been subjected to surgical operation in the reproductive system.

\section{Exclusion Criteria:}

Patients with ovarian failure undergoing oocyte donation.

\section{Methodology}

All patients attended the clinic for blood test and pelvic ultrasound examination at the beginning of the IVF treatment cycle before commencing ovarian stimulation.
Table 1: Characteristics of the pregnancy group vs. no pregnancy group (Age $\mathrm{AMH}$, number of retrieved oocytes, number of mature oocytes, number of embryos, FSH, AFC).

\begin{tabular}{|c|c|c|c|}
\hline Group & Pregnancy & No Pregnancy & P-Value \\
\hline Age & 32.05 & 32.1 & 0.968 \\
\hline AMH level (ng/mL) & 2.2148 & 1.2407 & 0.0002 \\
\hline Retrieved oocytes & 12.95 & 8.08 & 0.0012 \\
\hline Mature oocytes & 9.73 & 5.55 & 0.0003 \\
\hline Number of embryos & 6.5 & 3.75 & 0.0011 \\
\hline FSH level & 6.5448 & 10.6933 & 0 \\
\hline AFC level & 14.1 & 9.67 & 0.0054 \\
\hline
\end{tabular}

Clinical details of all treatment cycles were entered into a computerized database, which were retrieved for analysis.

Subjects were allowed to have replacement of at most two embryos two days' post-fertilization.

Embryo transfer (ET) was performed under trans-abdominal ultrasound guidance using a soft Catheter.

Fresh embryo transfer would be cancelled and all the embryos with good quality were cryopreserved on day 2 after the retrieval if the subject had symptoms suggestive of ovarian hyperstimulation syndrome (OHSS).

Finally, determination of pregnancy outcome was performed (urine pregnancy test, ultrasound examination later to confirm intrauterine pregnancy and the number of gestational sacs present).

\section{Results}

\section{Demographic analysis}

A total of 109 females who were seeking IVF at Mount Lebanon hospital fertility center were enrolled in the study, 9 patients were excluded for oocyte donation. The mean age was $32.08 \pm 6$ years, with a minimum of 14 years and a maximum of 44 years. Pregnancy rate was positive in 40 patients (40\%) and negative in 60 patients $(60 \%)$.

First, patients were distributed between two groups; pregnancy group and no pregnancy group and we have compared the IVF outcome (pregnancy indicated by urine Beta-HCG test) to mean age, serum AMH level, number of mature oocytes, number of embryos, FSH and AFC.

Characteristics like age, serum AMH, FSH, AFC, number of retrieved oocytes, number of mature oocytes and number of embryos are presented in Table 1.

In concern of age, the mean age of the pregnancy group was 32.05 years compared to 32.1 years in no pregnancy group, there is no significant difference, p-value 0.968 .

Our main result of interest is the distinction made by serum $\mathrm{AMH}$ concentrations between the two groups. This is demonstrated with the difference between means. Mean AMH in pregnancy group $(2.2148 \mathrm{ng} / \mathrm{ml})$ was higher than in no pregnancy group $(1.207 \mathrm{ng} / \mathrm{ml})$, p-value 0.0002 .

Same relation was found for number of oocytes and embryos, with p-value less than 0.01 . 
Table 2: Ovarian response of women undergoing IVF in relation to age, $\mathrm{AMH}$, number of mature oocytes, number of embryos, FSH and AFC. The increase in the mean levels of AMH paralleled the increase in the total number of oocytes, showing $0.555 \mathrm{ng} / \mathrm{ml}, 1.098 \mathrm{ng} / \mathrm{ml}, 2.171 \mathrm{ng} . \mathrm{ml}, 3.313 \mathrm{ng} / \mathrm{ml}$ in poor, normal, good and high responders respectively, with a p-value less than 0.01 .

\begin{tabular}{|c|c|c|c|c|c|}
\hline $\begin{array}{l}\text { Group } \\
(T=100)\end{array}$ & $\begin{array}{c}\text { Poor responders } \\
n=33\end{array}$ & $\begin{array}{c}\text { Normal responders } \\
n=22\end{array}$ & $\begin{array}{c}\text { Good responders } \\
n=25\end{array}$ & $\begin{array}{l}\text { High Responders } \\
n=20\end{array}$ & P-Value \\
\hline Age & $\begin{array}{c}31.394 \pm 2 \\
22-44\end{array}$ & $\begin{array}{c}33.318 \pm 2.939 \\
22-44\end{array}$ & $\begin{array}{c}31.56 \pm 2.409 \\
19-44\end{array}$ & $\begin{array}{c}32.5 \pm 3.349 \\
22-44\end{array}$ & 0.6602 \\
\hline $\mathrm{AMH}$ & $\begin{array}{c}0.555 \pm 0.13 \\
0.2-1.9\end{array}$ & $\begin{array}{c}1.098 \pm 0.282 \\
0.23-3\end{array}$ & $\begin{array}{c}2.171 \pm 0.371 \\
0.4-4.4\end{array}$ & $\begin{array}{c}3.313 \pm 0.536 \\
0.9-6\end{array}$ & $\begin{array}{c}1.58 \mathrm{E}-20 \\
(<0.01)\end{array}$ \\
\hline Number of mature oocytes & $\begin{array}{c}2.424 \pm 0.382 \\
0-4\end{array}$ & $\begin{array}{c}4.545 \pm 0.737 \\
7-J a n\end{array}$ & $\begin{array}{c}8.4 \pm 1.29 \\
0-14\end{array}$ & $\begin{array}{c}16.6 \pm 1.795 \\
\text { 24-Jun }\end{array}$ & $\begin{array}{c}2.88 \mathrm{E}-33 \\
(<0.01)\end{array}$ \\
\hline Number of embryos & $\begin{array}{c}1.667 \pm 0.432 \\
0-4\end{array}$ & $\begin{array}{c}3.318 \pm 0.745 \\
7-J a n\end{array}$ & $\begin{array}{c}6.48 \pm 1.286 \\
0-12\end{array}$ & $\begin{array}{c}9.75 \pm 2.118 \\
0-19\end{array}$ & $\begin{array}{c}8.52 \mathrm{E}-16 \\
(<0.01)\end{array}$ \\
\hline $\mathrm{FSH}$ & $\begin{array}{c}12.586 \pm 1.811 \\
1.36-23\end{array}$ & $\begin{array}{c}9.885 \pm 1.499 \\
4.6-17\end{array}$ & $\begin{array}{c}6.957 \pm 1.386 \\
1.96-15\end{array}$ & $\begin{array}{c}4.832 \pm 1.271 \\
1.9-14\end{array}$ & $\begin{array}{c}3.79 \mathrm{E}-09 \\
(<0.01)\end{array}$ \\
\hline AFC & $\begin{array}{c}5.697 \pm 1.491 \\
0-22\end{array}$ & $\begin{array}{c}8.864 \pm 1.607 \\
14-J a n\end{array}$ & $\begin{array}{l}14.2 \pm 2.398 \\
\text { Feb- } 29\end{array}$ & $\begin{array}{l}20.3 \pm 3.719 \\
\text { Feb-44 }\end{array}$ & $\begin{array}{c}8.60 \mathrm{E}-14 \\
(<0.01)\end{array}$ \\
\hline
\end{tabular}

Table 3: Correlations between AMH and FSH, AFC and Age.

\begin{tabular}{|c|c|c|c|}
\hline Correlations with AMH & FSH & AFC & Age \\
\hline P-Value & $8.00 \mathrm{E}-14$ & $2.00 \mathrm{E}-21$ & 1 \\
\hline
\end{tabular}

Table 4: Characteristics of the OHSS vs. no OHSS group (Age, AMH, number of retrieved oocytes, number of mature oocytes, number of embryos, FSH, AFC).

\begin{tabular}{|c|c|c|c|}
\hline Group & OHSS & No OHSS & P-Value \\
\hline Age & 30.67 & 32.12 & 0.684 \\
\hline AMH & 4.2377 & 1.5497 & 0.0004 \\
\hline Retrieved oocytes & 18.33 & 9.77 & 0.051 \\
\hline Mature oocytes & 13.33 & 7.03 & 0.066 \\
\hline Number of embryos & 7.67 & 4.76 & 0.241 \\
\hline FSH & 3.1667 & 9.2154 & 0.04 \\
\hline AFC & 22.33 & 11.1 & 0.015 \\
\hline
\end{tabular}

Table 5: Characteristics and distribution of patients from each group of serum AMH level on OHSS group and no OHSS group.

\begin{tabular}{|c|c|c|c|c|c|}
\hline \multicolumn{2}{|c|}{} & \multicolumn{2}{c|}{ OHSS } & \multirow{2}{*}{ Total } & \multirow{2}{*}{ P-Value } \\
\cline { 2 - 5 } & No OHSS & OHSS & & \\
\hline \multirow{3}{*}{ AMH groups } & {$[0-2]$} & 70 & 0 & 70 & \\
\cline { 2 - 5 } & {$[2.01-4]$} & 24 & 1 & 25 & \multirow{2}{*}{0.000003} \\
\cline { 2 - 5 } & {$[4.01-6]$} & 3 & 2 & 5 & \\
\hline \multirow{2}{*}{ Total } & & 97 & 3 & 100 & \\
\hline
\end{tabular}

Mean FSH in the pregnancy group (6.5448) was lower than in no pregnancy group (10.6933), with p-value less than 0.01 .

Mean AFC in the pregnancy group (14.1) was higher than in no pregnancy group (9.67), with a p-value 0.0054 .

\section{Ovarian response of women attending IVF program in relation to investigated parameters}

According to the number of oocytes retrieved upon stimulation by gonadotrophins, we have classified our patients into four categories:

- $\quad$ Poor responders ( $<4$ oocytes): 33 patients

- $\quad$ Normal responders (4-8 oocytes): 22 patients

- $\quad$ Good responders (9-16 oocytes): 25 patients

- High responders ( $>16$ oocytes): 20 patients

Characteristics and means of those 4 groups are presented in
Table 2.

We found the same relation with the number of retrieved oocytes and the number of mature oocytes and embryos, also with p-value less than 0.01 .

As the number of retrieved oocytes increases from poor to high responders, serum FSH declines (12.586; 9.885; 6.957; 4.832 for poor, normal, good and high responders respectively) with a p-value less than 0.01 .

As the number of retrieved oocytes increase from poor to high responders, AFC rises $(5.697 ; 8.864 ; 14.2 ; 20.3$ for poor, normal, good and high responders respectively) also with a p-value less than 0.01 .

We did not find a relationship between age of patients and number of oocytes retrieved, p-value 0.6602 , and this is probably because of the small number of patients in the study, and this result can be changed with larger population.

Women with high serum AMH level and AFC, low serum FSH, are more likely to respond to IVF stimulation as predicted by number of retrieved oocytes (Table 3).

The Spearman analysis was performed to detect the correlations between AMH and different outcomes of this study: there is significant association between $\mathrm{AMH}, \mathrm{FSH}$, and $\mathrm{AFC}$ but not between $\mathrm{AMH}$ and age.

Correlation between $\mathrm{AMH}$ and FSH showed a p-value less than 0.01 . The same result with AFC, but $\mathrm{p}$-value was 1 with age showing that those two variables are independent, and as we said above this result can be modified by increasing the number of patients recruited for the study (Figure 4-6).

From our 100 patients, only 3 females developed ovarian hyperstimulation syndrome. So we divided patients between two groups OHSS and no OHSS to analyze different parameters between females who had OHSS and females who did not. All the characteristics were presented in Table 4.

The only significant difference was the serum AMH level between the 2 groups. Mean serum AMH level was higher $(4.2377 \mathrm{ng} / \mathrm{ml})$ in the OHSS group than in no OHSS group (1.5497) with a p-value of 0.0004 .

AFC was higher in the OHSS group (22.33) than in no OHSS group (11.1) with a non significant p-value 0.015 . 
FSH was lower in OHSS group (9.2154) than in no OHSS group (3.1667) with a non significant $\mathrm{p}$-value of 0.04 .

Stressing on those results we divided patients into 3 groups regarding their serum AMH level. First group; patients with serum AMH level less or equal $2 \mathrm{ng} / \mathrm{ml}$, second group; patients with serum AMH level less or equal $4 \mathrm{ng} / \mathrm{ml}$, and third group; patients with serum AMH level more than $4 \mathrm{ng} / \mathrm{ml}$. results and distribution of each group between OHSS group and no OHSS group are presented in Table 5.

Results showed that none of the patients with serum AMH level less than $2 \mathrm{ng} / \mathrm{ml}$ had an ovarian hyperstimulation syndrome.

One of the 25 patients (4\%) with serum AMH level between 2 and $4 \mathrm{ng} / \mathrm{ml}$ has an OHSS.

Two of the 5 patients (40\%) with serum AMH level above 4ng/ $\mathrm{ml}$ had an OHSS.

These results were significant with a p-value less than 0.01 .

\section{Discussion}

Many factors have the potential to affect the outcome of the pregnancy during IVF/ICSI. Traditional parameters including FSH, E2 and INH-B are not entirely reliable. However, it has been proposed that estimation of $\mathrm{AMH}$ might provide an alternative approach given its high reproducibility and versatility allowing it to be checked at any time during the menstrual cycle.

Data presented in this study dealt with 100 women enrolled in IVF programs. By dividing patients into two groups, pregnancy and no pregnancy group, and comparing all parameters between the two groups, we found that serum AMH level is significantly higher in the pregnancy group than in no pregnancy group $(2.2148 \mathrm{ng} / \mathrm{ml} \mathrm{vs}$. $1.207 \mathrm{ng} / \mathrm{ml}$, p-value 0.0002 ), which confirms the positive correlation between serum AMH level and positive pregnancy.

Also, a positive correlation was found with mean AFC (14.1 vs. 9.67, p-value <0.01) confirming that an increase in antral follicle count increases the probability of a positive pregnancy in females undergoing there first IVF cycle. Another relation between FSH level and pregnancy confirmed that low FSH level correlates with a positive pregnancy (6.5448 vs. 10.6933, p-value< 0.01).

These results presented in our study confirm that serum AMH level is an equivalent marker to FSH and AFC in predicting positive outcome in females undergoing IVF.

Several investigators have reported similar successive increase in the total number of oocytes as a result of increasing $\mathrm{AMH}$ levels in responder women attending IVF.

In a study that aims to evaluate whether serum anti-Müllerian hormone measurement on any day of the menstrual cycle strongly predicts ovarian response in assisted reproductive technology, blood withdrawal for AMH measurement was performed in all the patients independently of the day of the menstrual cycle and showed that women in the lowest AMH quartile $(<0.4 \mathrm{ng} / \mathrm{ml})$ were older and required a higher dose of recombinant FSH than women in the highest quartile $(>7 \mathrm{ng} / \mathrm{ml})$. All the cancelled cycles due to absent response were in the group of the lowest AMH quartile, whereas the cancelled cycles due to risk of ovarian hyperstimulation syndrome
(OHSS) were in the group of the highest AMH quartile. This study demonstrated a strong correlation between serum AMH levels and ovarian response to gonadotrophin stimulation. This study showed that $\mathrm{AMH}$ is a reliable serum marker of ovarian response that can be measured independently of the day of the menstrual cycle [23].

Another study performed to investigate whether anti-Müllerian hormone and antral follicle count can be useful in predicting the ovarian reserve and pregnancy outcome in assisted reproductive technology cycles showed that there was an association between the serum level of anti-Müllerian hormone in early follicular phase and ovarian reserve. Furthermore, a higher serum level of anti-Müllerian hormone on day three was associated with chemical pregnancy success [24].

Another prospective study on women undergoing their first IVF/ ICSI cycle showed significant differences between females with less than 4 oocytes and females with more than 4 oocytes regarding FSH $(\mathrm{p}=0.019)$, baseline AMH $(\mathrm{p}=0.002), \operatorname{AFC}(\mathrm{p}<0.001)$, day $5 \mathrm{AMH}$ $(\mathrm{p}=0.005)$, but not for follicular AMH $(\mathrm{p}=0.183)$; which proves that baseline AMH is almost as good as a predictor for poor ovarian response as AFC [25].

In our study, age between the two groups was not significantly different (32.05 years vs. 32.1 years, p-value 0.968 ) which showed that the chance of IVF success was not increased with decreased age as predicted and demonstrated in previous studies.

This dissociation between age and success of IVF showed in our study may be due to the small number of patients. Further studies with larger numbers of women are required to confirm the previous published articles.

Previous studies showed that younger women had a better chance to have a successful pregnancy. Retrospective study that evaluates relationship between serum $\mathrm{AMH}$ and the rate of ongoing pregnancy in first embryo transfer cycles showed that serum AMH measurements reflect the total pool of AMH-producing follicles. Serum AMH level is closely related to the ovarian reserve and might be of secondary importance for ongoing pregnancy, with the most important factor for pregnancy being age [18].

Stressing on number of retrieved oocytes, we have classified our patients into four categories; poor, normal, good and high responders according to number of retrieved oocytes $(<4,4$ to 8,9 to 16 and $>16$ respectively) [26].

Analyses of data collected from each group showed that the mean levels of serum AMH level showed a progressive increase $(0.555 \mathrm{ng} /$ $\mathrm{ml}, 1.098 \mathrm{ng} / \mathrm{ml}, 2.171 \mathrm{ng} / \mathrm{ml}$ and $3.313 \mathrm{ng} / \mathrm{ml}$ ) parallel to the number of retrieved oocytes in poor, normal, good and high responders, with a p-value less than 0.01.This means that high mean levels of AMH can be linked to the increased mean number of collected oocytes and consequently to increased chance of obtaining higher number of embryos improving the chance of pregnancy. This confirms that serum AMH level is a good indicator for ovarian response as FSH, AFC and Age.

AFC also showed a positive correlation with number of retrieved oocytes (5.697, 8.864, 14.2 and 20.3 respectively for poor, normal, good and high responders, p-value $<0.01$ ). 
And FSH level decreases with increased number of retrieved oocytes $(12.586,9885,6.957$ and 4.832 with a p-value $<0.01)$.

To confirm the relation of serum AMH level and different ovarian reserve markers, we performed Spearman analysis to detect correlations between serum AMH level and AFC, FSH and Age. This correlation was confirmed with FSH (p-value $<0.01$ ), AFC (p-value $<0.01$ ) and not with patients' Age (p-value 1) and this was because of the small number of patients recruited.

Ovarian hyperstimulation syndrome, a main concern to prevent for all gynecologist by balancing between hormonal stimulation and ovarian response of every patient, was our second point of interest.

In our study only 3 of 100 patients developed hyperstimulation ovarian syndrome. By comparing different parameters of each group of patients, group with OHSS and group with no OHSS, we found that high serum AMH level had the great correlation $(4.2377 \mathrm{ng} / \mathrm{ml}$ vs. $1.5497 \mathrm{ng} / \mathrm{ml}$, p-value 0.0004 ) with OHSS.

This correlation was superior to that found with AFC (22.33 vs. 11.1, p-value 0.015 ) and FSH (3.1667 vs. 9.2154, p-value 0.04) (Table 4).

So higher level of AMH was associated with increased risk of developing hyperstimulation ovarian syndrome in response to IVF drugs as mentioned in previous studies (Mean AMH levels in serum and in FF were significantly higher in patients with OHSS than in normal patients) [21].

Stressing on these results, we divided our patients into three groups according to serum AMH level; first group AMH level less than $2 \mathrm{ng} / \mathrm{ml}$, second group AMH level between $2 \mathrm{ng} / \mathrm{ml}$ and $4 \mathrm{ng} / \mathrm{ml}$ and third group AMH level more than $4 \mathrm{ng} / \mathrm{ml}$ (Table 5).

Analyses of data showed that none of the patients of the first group had OHSS, $4 \%$ of patients of the second group had OHSS and $40 \%$ of patients of the third group had OHSS (p-value $<0.01$ ). This confirms that the risk of developing ovarian hyperstimulation syndrome increases exponentially with the increase of serum AMH level, with a level above $4 \mathrm{ng} / \mathrm{ml}$ being more likely to induce OHSS.

\section{Conclusion}

Data collected in our study confirms the validity of serum AMH level as a marker for ovarian reserve and ovarian response during IVF, with an equivalent benefit to this acquired with other markers like FSH, AFC and Age.

Also serum AMH level confirmed to have a superiority on other markers in detecting occurrence of ovarian hyperstimulation syndrome. Women with a high AMH level are likely to respond excessively to exogenous gonadotrophins and their treatment strategy can be modified to minimize the risk of ovarian hyperstimulation syndrome (OHSS), in contrast to women with a low AMH, who are likely to respond poorly to stimulation with consequently a low chance of pregnancy, and their expectations can be managed appropriately with alternatives like oocyte donation.

Further studies with a larger population and including different medical situations like polycystic ovary syndrome and his correlation with serum AMH level are in our goals to confirm the use of this marker for diagnosis of PCOS and to confirm the correlation between age and serum AMH level.

\section{References}

1. Hull M, Glazener C, Kelly NJ, Conway DI, Foster PA, Hinton RA, et al. Population study of causes, treatment, and outcome of infertility. BMJ. 1985; 291: 1693-1697.

2. Scott RT, Osaphl MS, Leonardi MR, Neall GS, Illions EH, Navot D. Life table analysis of pregnancy rates in a general infertility population relative to ovarian reserve and patient age. Hum Reprod. 1995; 10: 1706.

3. Van der Linden M, Buckingham K, Farquhar C, Kremer JAM, Metwally M. Luteal phase support for assisted reproduction cycles. Cochrane Database of Systematic Reviews. 2011; 10.

4. Grossman M, Nakaji S, Fallat $\mathrm{M}$, et al. Müllerian inhibiting substance inhibits cytochromeP450 aromataze activity in human granulosa lutein cell culture. Fertil Steril. 2008; 89: 1364-1370.

5. Lie Fong S, Visser JA, Welt CK, et al. Serum anti-müllerian hormone levels in healthy females: a nomogram ranging from infancy to adult- hood. J Clin Endocrinol Metab. 2012; 97: 4650-4655.

6. Tal R, Seifer DB. Potential mechanisms for racial and ethnic differences in antimüllerian hormone and ovarian reserve. Int J Endocrinol. 2013; 81: 8912.

7. Iliodromiti S, Kelsey TW, Anderson RA, et al. Can anti-Mullerian hor- mone predict the diagnosis of polycystic ovary syndrome? A systematic review and meta-analysis of extracted data. J Clin Endocrinol Metab. 2013; 98: 33323340.

8. Nastri CO, Ferriani RA, Rocha IA, Martins WP. Ovarian hyperstimulation syndrome: pathophysiology and prevention. J Assist Reprod Genet. 2010; 27: $121-128$.

9. Aboulghar MA, Mansour RT. Ovarian hyperstimulation syndrome: classifications and critical analysis of preventive measures. Hum. Reprod. 2003; 9: 275-289.

10. Yates AP, Rustamov O, Roberts SA, Lim HYN, Pemberton PW, Smith A, et al. Anti-Mullerian hormone-tailored stimulation protocols improve outcomes whilst reducing adverse effects and costs of IVF. Hum Reprod Oxf Engl. 2011; 26: 2353-2362.

11. La Marca A, Argento C, Sighinolfi G, Grisendi V, Carbone M, D'Ippolito G, et al. Possibilities and limits of ovarian reserve testing in ART. Curr Pharm Biotechnol. 2012; 13: 398-408.

12. Safier LZ, Grossman LC, Chan CW, Sauer MV, Lobo RA, Douglas NC. Use of anti-Müllerian hormone testing during ovarian reserve screening to identify women at risk of polycystic ovary syndrome. Int J Gynaecol Obstet Off Organ Int Fed Gynaecol Obstet. 2016; 135: 73-76.

13. Jamil Z, Fatima SS, Ahmed K, Malik R. Anti-Mullerian Hormone: Above and Beyond Conventional Ovarian Reserve Markers. Dis Markers. 2016; 2016: 5246217.

14. van Disseldorp J, Faddy MJ, Themmen APN, de Jong FH, Peeters PHM, van der Schouw YT, et al. Relationship of serum antimüllerian hormone concentration to age at menopause. J Clin Endocrinol Metab. 2008; 93: 2129-2134.

15. Depmann M, Eijkemans MJC, Broer SL, Scheffer GJ, Laven JSE, et al. Does anti-Müllerian hormone predict menopause in the general population? Results of a prospective ongoing cohort study. Hum Reprod Oxf Engl. 2016; 31: 1579-1587.

16. Anderson RA, Cameron DA. Pretreatment serum anti-müllerian hormone predicts long-term ovarian function and bone mass after chemotherapy for early breast cancer. J Clin Endocrinol Metab. 2011; 96: 1336-1343.

17. Wiweko B, Prawesti DMP, Hestiantoro A, Sumapraja K, Natadisastra M, Baziad A. Chronological age vs biological age: an age-related normogram for antral follicle count, FSH and anti-Mullerian hormone. J Assist Reprod Genet. 2013; 30: 1563-1567.

18. Honnma H, Baba T, Sasaki M, Hashiba Y, Oguri H, Fukunaga T, et al. Serum 
anti-Mullerian hormone levels affect the rate of ongoing pregnancy after in vitro fertilization. Reprod Sci Thousand Oaks Calif. 2013; 20: 51-59.

19. Lukaszuk K, Kunicki M, Liss J, Lukaszuk M, Jakiel G. Use of ovarian reserve parameters for predicting live births in women undergoing in vitro fertilization. Eur J Obstet Gynecol Reprod Biol. 2013; 168: 173-177.

20. Kunt C, Ozaksit G, Keskin Kurt R, Cakir Gungor AN, Kanat-Pektas M, Kilic $\mathrm{S}$, et al. Anti-Mullerian hormone is a better marker than inhibin $\mathrm{B}$, follicle stimulating hormone, estradiol or antral follicle count in predicting the outcome of in vitro fertilization. Arch Gynecol Obstet. 2011; 283: 1415-1421.

21. Salmassi A, Mettler L, Hedderich J, Jonat W, Deenadayal A, von Otte S, et al. Cut-Off Levels of Anti-Mullerian Hormone for The Prediction of Ovarian Response, In Vitro Fertilization Outcome and Ovarian Hyperstimulation Syndrome. Int J Fertil Steril. 2015; 9: 157-167.

22. Grynnerup AG-A, Lindhard A, Sørensen S. The role of anti-Müllerian hormone in female fertility and infertility - an overview. Acta Obstet Gynecol Scand. 2012; 91: 1252-1260.
23. La Marca A, Giulini S, Tirelli A, Bertucci E, Marsella T, Xella S, et al. AntiMüllerian hormone measurement on any day of the menstrual cycle strongly predicts ovarian response in assisted reproductive technology. Hum Reprod Oxf Engl. 2007; 22: 766-771.

24. Dehghani-Firouzabadi R, Tayebi N, Asgharnia M. Serum level of antimullerian hormone in early follicular phase as a predictor of ovarian reserve and pregnancy outcome in assisted reproductive technology cycles. Arch Iran Med. 2008; 11: 371-376.

25. Tolikas A, Tsakos E, Gerou S, Prapas Y, Loufopoulos A. Anti-Mullerian Hormone (AMH) levels in serum and follicular fluid as predictors of ovarian response in stimulated (IVF and ICSI) cycles. Hum Fertil Camb Engl. 2011; 14: 246-253.

26. Kumar P, Sait SF, Sharma A, Kumar M. Ovarian hyperstimulation syndrome. J Hum Reprod Sci. 2011; 4: 70-75.
Austin J Obstet Gynecol - Volume 5 Issue 6 - 2018

Submit your Manuscript | www.austinpublishing group.com

Darido et al. (C) All rights are reserved
Citation: Moubarak H, Mokdad M, Farhat S, Fakih C and Darido J. Correlation between Serum Anti-Müllerian Hormone (AMH) Level in Women and Follicular Response in the First In Vitro Fertilization (IVF) Cycle in Predict Live Birth from One Stimulation Cycle. Austin J Obstet Gynecol. 2018; 5(6): 1117. 